\title{
Alención Médica de la Embarazada Tuberculosa
}

\author{
Doctor Luis F. García \\ Obstetra del Hospital Santa Clara
}

El siguiente estudio, presentado a la Primera Convención Nacional de Obstetricia y Ginecología, es el resultado de cuidadosas consultas bibliográficas y de observaciones personales hechas en el servicio de maternidad, a mi cargo, en el Hospital-Sanatorio de Santa Clara.

Conviene hacer una breve descripcion de las modificaciones que sufre el varato respiratorio de la gestante normal, la influencia del embarazo sobre la

B.C. pulmonar y viceversa y la accion de la misma entidad nosológica sobre i ieto.

En los primeros meses del embarazo no hay cambio apreciable en el apa1.atu respiratorio; en la segunda mitad del embarazo, los diámetros ante-posteriotis ran disminuyendo gradualmente, teniendo una compensación en el mayor unanchamiento de la caja torácica. Asi mismo el campo respiratorio disminuye prir el desp'azamiento, gradual también, que sufre el diafragma hacia arriba; poro esto no altera la hematosis, pues la mavor frecuencia respiratoria compensa hi menor cantidad de aire que entra en cada inspiración. De ahí la falsa disnea the experimenta la mujer que se halla en el ultimo período de la gestación y disminuve sobre todo en la primigestante hacia el octavo mes, cuando por ncajamiento de la presentacion el diafragma desciende.

La circulación del aire, comọ la sanguinea, en el interior del pulmón, tien mayor actividad durante el embarazo que fuera de él. Existe un aumento i. la eliminación del ácido carbonico en las gestantes por estar en ellas aumenwdus las combustiones orgánicas.

Imholer (1), hizo notar en 1910 la frecuencia con que aparecen tumefact.1. las cimas pulmonares en las embarazadas; este hecho debe ser tenido en cuent. para no atribuír tal fenómeno fisiolcgico a un proceso tuberculoso.

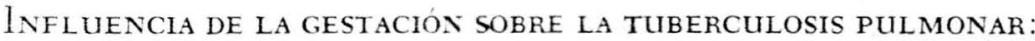

La manera de comportarse el organismo materno frente a una tuberculosis pulmonar, ha tenido varias apreciaciones. Hasta hace aproximadamente un si(2h). los más afamados clínicos atribuían una cierta acción favorable del em- 
barazo sobre la T.B.C. pulmonar. Ciefto tiempes después, Louis y Grisolle opinar in que se encontraba alguna agravacion de las lesiones tuberculosas en las mujeres que aparecían embarazadat, fue thitnces cuando resolvieron suprimir la causa de tal agravación y empezh, a adeptarse como un dogma la conducta del aborto terapéutico. Hoy día, las upinimés estan divididas contando con más adeptos, una corriente intermedia que as zutd, que, si el embarazo puede causar algún efecto nocivo sobre la tule tutheric, tate se puede contrarrestar por medio de los nuevos tratamientos que $\psi$ han veaclo para esta enferma. Lo que sí es cierto, es que la gestación afcen methemente las formas avanzadas de la tuberculosis, dependiendo por lo tatin di lifk de lesion tuberculosa y del momento de aparición en relación con el tmbaram.

La agravacion de la tubercules: fret el embarazo se puede clasificar de la siguiente manera $\left({ }^{2}\right)$ :

10-Las tuberculosis benigna: " Ntriatas " que mediante el tratamiento han evoluciunado hacia la esclerosis tentencondr, hevolución; estas formas sólo se agravan en el $8 \%$ de los casos.

20-Las formas úlcero-caseosa: "wn fremas únicas o múltiples localizadas en uno o en ambos pulmones; esta: fimas syavan siompre con el embarazo; dentro de este grupo, Sergent ha hat he rif suligrupt en el cual incluye las for-

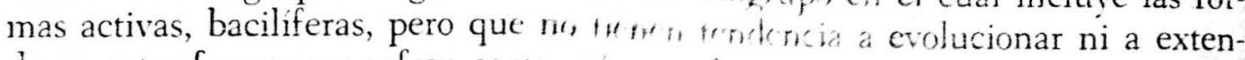
derse; estas formas no sufren agraviatu wo h gestacion.

30-Las pleuresias y congestim prulmomires de origen tuberculoso; éstas

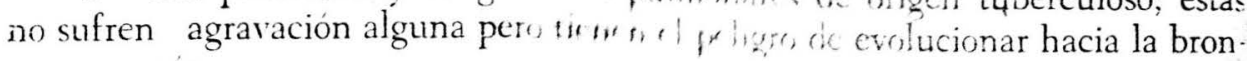
coneumonía.

$4^{\circ}$ - La forma que sufre mayor ayt in in de pronistico más desfavorable es la tuberculosis laríngea pues se the rvan ytance crisis disneicas que ponen en peligro la vida de la enferma.

En resumen, las formas inactwa y y mantiestan ninguna agra

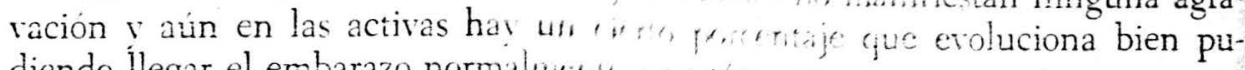

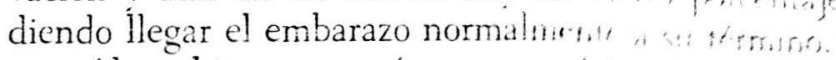

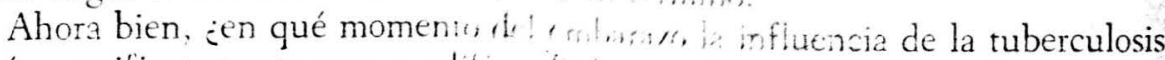
es más manifiesta tanto para modifu... f frema tinica como para el pronóstico de la afección?

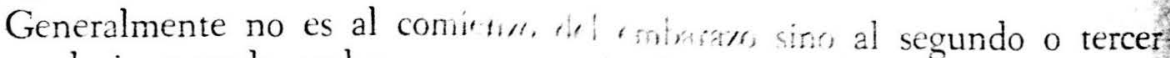

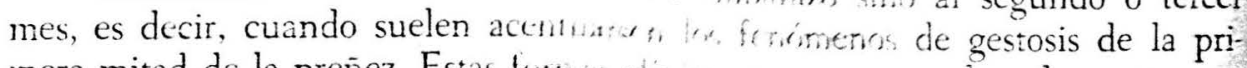
mera mitad de la preñez. Estas form en furesponden al aparato gastro-intestinal, como son los vúmiks were fomplo, agravan en seguida

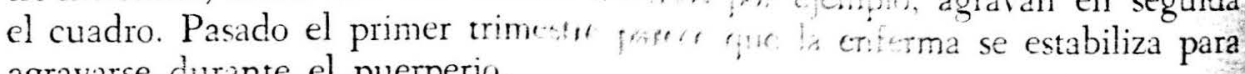
agravarse durante el puerperio.

Tapia ( ${ }^{3}$, analiza tres grupus it thros gue ha observado; el primer grupo lo constituyen enfermas con lew. fusculosar que datan de algún

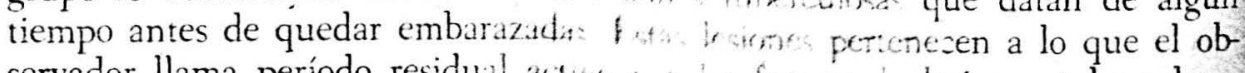
servador llama período residual actor,

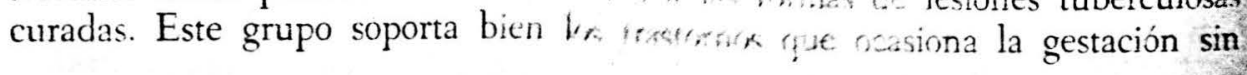


manifestar signos de actividad. Sin embargo, en un pequeño número ha observado ligeras alzas térmicas, astenia, anorexia, etc., fenómenos vagos que no dan a pensar que se trate de un brote evolutivo. También es necesario aclarar que la agravación está en razón inversa con el tiempo a que se remontan los brotes tuberculosos anteriores, de tal manera que es frecuente ver la evolución normal del embarazo, parto y puerperio, en enfermas que años antes habían tenido episodios de hemoptisis, disnea, fiebre, etc.

El segundo grupo está integrado por enfermas con lesiones tuberculosas en actividad y quedan embarazadas. Tapia lo divide en tres subgrupos.

a) Formas preferentemente caseosas; de una manera general, las enfermas pertenecientes a este subgrupo se ven empeoradas gradualmente por el embarazo hasta llegar a la muerte; tratadas convenientemente pueden tolerar bien el cmbarazo y parto pero no el puerperio.

b) Formas iniciales y fibro-caseosas que tienen dos variedades: en una, ve mos que la gestación influve de una manera francamente nociva aumentando la capacidad invasora del proceso; y en otra parece que el embarazo no afecta mavormente la evolucion de la enfermedad demostrando las enfermas una gran resistencia. Incluso es frecuente notar que, a partir del cuarto o quinto mes las c nfermas presentan una mejoría más o menos notoria que la mayoría de los autoatribuyen al ascenso del diafragma.

c) Formas fibro-caseosas avanzadas y cirróticas; en las primeras, el embarazo welera la muerte, $\mathrm{y}$ en las cirróticas. si bien a veces toleran la gestación, en cambio el parto constituve un grave peligro por el déficit circulatorio que existe en cistas formas.

En la práctica sin embargo, es extremadaménte raro que mujeres con lesiones caseosas evolutivas. queden embarazadas, pues las transformaciones estructurales que sufren las trompas a consecuencia de las anexitis secundarias, producen una esterilidad definitiva.

Por fin, el tercer grupo es el que pudiéramos llamar, de tuberculosis gravídica, ves está formado por aquellas tuberculosis que aparecen por primera vez dur.nte el embarazo. Esta forma esta caracterizada por lesiones infiltrativas, ya xa del grupo del infiltrado precoz, ya del grupo denominado post-hematógeno. Evolucionan en forma distinta: unas veces evolucionan bien siempre y cuando descle el momento de su aparicion se hava instalado una terapéutica colapsante, $\checkmark$ otras veces, las formas se vuelven agudas agravando el pronóstico Pero en general, según Sergent ( $\left.{ }^{+}\right)$, la tuberculosis que se inicia durante el embarazo sucle llevar una evolución acelerada, presentando más gravedad en la primípara sobre todo cuando es joven.

Las etapas que más influven en la agravación de la T.B.C. son el parto y ecialmente el puerperio. La fatiga y esfuerzo del parto, al congestionar el pulmón, pueden provocar hemoptisis fulminantes. En la puérpera, una T.B.C. atente o de curso lento se agrava con brusquedad. Se puede observar un reblandecimiento rápido del pulmón, brotes de bronconeumonía tuberculosa que con-

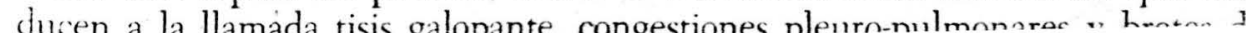


granulia, o bien se produce una verdadera septicemia tuberculosa rápidamente mortal (meningitis). Se ha mencionado la muerte súbita después del parto, cuan do se presenta sínfisis pleural o granulaciones miliares de la pleura.

Durante el puerperio muchas enfermas sucumben; y ésto sucede no sólo entre las que se agravaron durante el embarazo, sino también entre las que se mantuvieron aparentemente en buenas condiciones. Asimismo, suele observarse la acentuación tardia de la enfermedad, en una época más o menos alejada del puerperio. La descompresión brusca del tórax después del parto, puede producir nuevas siembras y por lo tanto, nuevos focos tuberculosos, poi la aspiración de gérmenes por las vías linfática y hemática. A este respecto, se ha llegado a pensar en un desgarro del tejido conjuntivo que rodea los focos tuberculosos, dando salida a los bacilos hasta entonces encarcelados (").

También se han invocado causas agravantes de cuya aparición se responsabiliza al parto. Por razón de su enfermedad, la mujer en trance de dar a luz, es débil y por lo tanto no está en condiciones de soportar una función que requiere el máximo de vitalidad, pudiendo llegar a la inercia uterina por agotamiento. Otra causa de agravación que hay que temer en el post-alumbramiento es la hemorragia uterina que termina de debilitar el organismo materno va agotado por un trabajo de parto.

Cuando toleran bien el embarazo y el parto la enfermedad se agrava en el cuarto o quinto dia del puerperio, así puede verse la aparición de temperaturas resperales hasta de 40 grados, taquicardia, anorexia, enflaquecimiento y al examen radiológico se observa el aumento de tamaño de las lesiones y la aparición de otras nuevas. Otras veces no es tan precoz esta agravación y sólo se hace en la segunda o tercera semana del puerperio. Sergent $\left({ }^{2}\right)$ considera como límite de agravación de la tuberculosis, en las mujeres que han dado a luz sin complicaciones, un año, tiempo durante el cual hay que vigilarlas permanentemente para tratarlas con oportunidad.

Las causas por las cuales influve en forma perjudicial el embarazo sobre la T.B.C., no son dificiles de comprender; juegan papel importante varios factores. Entre ellos, mencionamos:

10-El factor metabćlico. Flovd $\left({ }^{3}\right)$, que hace resaltar este factor, al com probar la exigencia que el embarazo hace del organismo traducido en un mayor consumo de yodo, hierro y calcio, concluye en que el metabolismo basal es un $30 \%$ más acelerado que normalmente y como consecuencia de ello se aumenta la función respiratoria.

$2^{\circ}$-Algunos autores (Hofbaber, Herman y Neuman $(5)$, han descrito una disminución del poder lipolitico del suero de las embarazadas y un aumento de las grasas en la sangre de las mismas. Como resultado de estos dos fenómenos se deduce que, en la embarazada se encuentra una disminución de la capacidad lipolitica para atacar la cubierta cérea que protege al bacilo de Koch. Experimentalmente se ha comprobado que en las tuberculosas hay una baja de la lipasa san 
guinea y Joshida hace hincapié en la utilidad pronóstica, en sentido benéfico, que tienen la dosificación de las lipasas del suero para comprobar si reaparecen durante el puerperio.

39-Nubiola-Zárate $\left(^{6}\right)$, sostienen que el embarazo es un estado tóxico en el cual el organismo materno reacciona, defendiéndose de las albúminas heterólogas de origen paterno; por lo tanto el embarzo es un estado de equilibrio bastante inestable. Además existe una notable insuficiencia de las glandulas endocrinas produciendo por consiguiente una alteración de todos los procesos nuiritivos.

4․-En la gestante hay un estado de anergia, es decir, de flaqueamiento de la inmunidad sobre todo en la última época de la preñez y comienzo del puerperio, comprobada por la atenuación e incluso desaparición de la cutirreacción a la tuberculina, en ese momento.

5- El hipertiroidismo que existe en el embarazo, es otro factor que agrava 1) proceso tuberculoso.

$6^{\circ}$ - El alza de la colesterinemia del embarazo es otra causa perjudicial en .1 to grado para la tuberculosis. De tal manera que tiene también un valor proustico, la tasa de la colesterina sanguínea que indica de qué manera puede olucionar la enfermedad.

7.-En la gestante hay una movilización de las reservas de calcio en prowho de la osificación del embrión. Botella (i) dice, que no sólo se moriliza A calcio óseo sino también, en las tuberculosas, se moviliza el de algunas lesiones calcificadas dejando por consiguiente en libertad a los bacilos de Koch.

8?-Un factor que agrava la T.B.C., descubierto en los últimos años, es I de la hormona estrógena; su mecanismo es sencillo: es sabido que en la embarazada hay una gran producción de estrógenos excitando en el organismo todos ln procesos de naturaleza alérgica y entre ellos la tuberculosis; se comprueba orque en las tuberculosas hay alzas apreciables de la temperatura en los dias Le preceden a la menstruación y por qué la enfermedad ataca con más vehe ncia a las mujeres con hiperfunción ovárica.

9. - Por último, un factor de agravación particular, es de orden mecánico. Sabido es, que durante el embarazo hay una inmovilizacion del parenquima pulmonar y menor expansión respiratoria. Pues bien: durante el parto ocurre una gran descompresión torácica recuperando los pulmones su volumen normal con la consiguiente agravación del proceso tuberculoso: aspiración en los bronquios de drenaje, diseminación hematógena, granulia y muertt.

\section{INfluencia de la tuberculosis pulmonar sobre el embarazo:}

Segun algunos autores se aprecia un gran efecto desfavorable, traducido en ia proporción de abortos a consecuencia de la tuberculosis. Estos dividen las formas clínicas de la T.B.C. en relación con el embarazo, en los 4 grupos siguientes ( $(\mathbf{l})$ : 
10-Formas inactivas en las cuales no se aprecia acción alguna sobre la ges tación; se menciona el hecho, de que aparece el aborto en una proporción de $5,3 \%$. es decir, una cifra inferior a la que se presenta en las gestantes normales.

2?-Formas infiltrativas no bacilares: en éstas el aborto se produce en una proporción del $6.4 \%$, cifra también inferior que la que aparace en las embara. zadas normales.

30-Formas bacilares localizadas en las que se presenta un 12 a $14 \%$ de abortos, cifra ya más alta que la que se encuentra en las gestantes normales que es de un 8 a $10 \%$

$4^{\circ}$ - Formas graves diseminadas que son las que dan el índice más alto de abortos, pues es del $50 \%$.

En proporción semejante se presentan los partos prematuros entre los 7 y 8 meses. También es alto el indice de partos distócicos.

Respecto de los porcentajes citados, tomados de Botella (T), creemos poco lógico que sea menor el riesgo de aborto para las madres tuberculosas que para las sanas. Esto podría explicarse por una desigualdad en los números de observaciones correspondientes a los dos grupos gestantes.

\section{INFLUENCIA SOBRE EI. PARTO Y PUERPERIO:}

Durante el parto se puede encontrar hipocontractilidad uterina que se tra duce en un trabajo largo y poco intenso; la causa de este fenómeno se atribuye al agotamiento y debilidad general de la tuberculosa. Asi mismo, durante el puerperio puede haber subinvolucion uterina que produce pequeñas pero constantes hemorragias que no deben ser menospreciadas.

\section{INFLUENCIA SOBRE EL FETO:}

Respecto a la tuberculosis congenita hav disparidad de criterios. Algunos autores sostienen que sí se verifica el contagio de la madre al hijo en la vida intrauterina y otros aseguran, que ésto no ozurre practicamente nunca. Segúr. los primeros, el feto se infectaria dé las siguientes maneras:

a) Por llevar el espermatozoide o el ovulo, el bacilo, la infección tendría lugar en el momento mismo de la fecundacion. Esto no se ha demostrado (7).

b) La infección del feto se efectuaria a partir de una lesion tuberculosa previa del útero y clementos ovulares. Segun Goldberg $(>$ ), esta lesión puede ser: 10-Endometritis tuberculosa que puede existir desde antes de comenzar el embarazo.

20-Una inflamación de la decidua basal (deciduitis), la cual es manifestada por formaciones tuberculosas localizadas $y$ ocasionalmente por una infiltración tuberculosa difusa.

30-Posiblemente han sido vistos bacilos en placentitis tuberculosa, lo mismo que tubérculos en las vellosidades coriales y bacilos en las células de Langhans. Doderlein $\left({ }^{9}\right)$, ha observado también la infección placentaria y opina 
Q en la mayoría de los casos, se afecta primeramente la cara materna de la placenta, localizándose el proceso tuberculoso en el espacio intervelloso o en la decidua basal.

Del espacio intervelloso, el bacilo alcanza el epitelio de las veliosidades produciendo, unas veces, necrosis de la rellosidad, trombosis y migración leucocitaria y otras veces formando un tubérculo de células gigantes. En la decidua basal se forman focos caseosos que van luégo a infectar la placenta fetal.

c) En los últimos años, parece que se ha demostrado el paso, a través de la vlacenta, de las formas filtrables del bacilo de Koch, visibles al ultramicroscopio. Se cree que estas formas no son capaces de producir tubérculos típicos ni en la placenta ni en los elementos ovulares pues son fases degenerativas del bacilo. Sobre el feto, su acción se limitaría a producir un cierto estado de impregnación tóxica que dificultaría su nutrición y sería la responsable de la gran debilidad - los niños, hijos de madres tuberculosas.

Las lesiones placentarias, pueden ser determinadas de distintos modos (1):

a) Los bacilos tuberculosos pueden ser llevados al feto, por la sangre materna, en los casos de T.B.C. miliar.

b) La pre-existencia de una lesión tuberculosa localizada en la mucosa utena, en el sitio en que se va a implantar el huevo, infectará la placenta, por connuidad de tejido.

c) Los coitos consecutivos al comienzo de la gestación, con individuos que icnen lesiones tuberculosas en sus genitales, producirían una inoculación directa de la infección.

Según Moenckerberg $\left(^{2}\right)$, hay varias formas de tuberculosis congénita, las cuales están bien clasificadas:

19-Forma bacilar y anatómica con tuberculosos típicos, que se pueden lo- lizar en el peritoneo, cápsulas suprarrenales, pulmón, hígado, bazo y ganglios mesentéricos. Se han descrito también, tubérculos en la frente y un tipo de pénzo-plantar, semejante al del heredo-luético, encontrándose el bacilo en dichas - ciones cutáneas.

$2^{\circ}$ - Bacilosis congénita sin lesiones anatémicas de ningún órgano pero con presencia del bacilo en la sangre.

39--Virulemia tuberculosa sin tubérculos anatómicos ni estado septicémico, pero con las formas filtrantes del bacilo en la sangre del niño, lo cual se prueba; porque al inocular a un curí, la sangre del cordón, se tuberculiza el animal, con aparición de grandes adenopatías y bacilos en los ganglios. Los niños comprendidos en esta forma de T.B.C. congénita, no tienen signos clínicos de tubercuhisis, pero son niños hipotrépsicos, desnutridos, en los cuales más tarde, en un mite que puede variar hasta la pubertad, hace su aparición la tuberculosis, aún - Aiéndolos tenido en el más riguroso aislamiento para descartar el contagio pust-natal.

Küss $\left({ }^{t}\right)$, sostiene que la herencia del terreno es lo más corriente; las toxinas pasan de la madre al feto, quedando éste predispuesto para contraer la infección 
después del nacimiento; con lo cual está de acuerdo Landouzzi ( ${ }^{4}$ ), al afirmar que el feto nace tuberculizable y no tuberculoso.

En la mayoria de los niños nacidos de tuberculosas, se aprecia un peso inferior al normal siendo el promedio de 2.700 gmos. en las formas leves y 2.000 gmos-, en las graves.

La rata de mortalidad de los niños nacidos de madres tuberculosas, es alto. Pankow y Küpferle $(x)$, han encontrado un $45,5 \%$, antes del primer año. Este alto porcentaje de mortalidad sería debido a que en su mayoria, estos niños presentan una inferioridad constitucional, según supone De Lee ('").

\section{Proxóstico:}

Por regla general los datos que nos indican el curso de la T.B.C. en la embarazadas, son los mismos que se encuentran en las enfermas no gestantes. La prueba de la tuberculina tiene valor pronóstico, teniendo en cuenta que la tasa de anticuerpos defensivos se encuentra disminuida durante el embarazo.

\section{Conducta y tratamiento}

El obstetra tiene una gran responsabilidad frente a una cmbarazada tuberculosa ya que cualquier falla en el tratamiento ensombrece el pronóstico.

Las medidas que se deben tomar son de dos clases: generales y especiales.

Durante la gestación se impone el intemamiento en $\in$ el sanatorio donde va a recibir la doble atención del tisiologo $\mathrm{y}$ del obstetra. Permitiéndose el tratamiento ambulatorio sólo en casos especiales de enferma que presenten lesiones incipientes.

Debe observarse una vigilancia cuidadosa en lo referente a dieta procurando sobrealimentar a la enferma, evitando el recargo exageradr que podría constituír un peligro para el embarazo; procuraremos manteneme iempre en las cifras de 85 a 90 gmos. diarios de proteinas. 50 a $60 \mathrm{gmm}$ de grasas y forzando los hidrocarbonados: no olvidaremos los factores accesnis. vitaminas y sales. Administraremos vitamina $\mathrm{D}$ en dosis altas parà producir el chrorue vitamínico; calcio en dosis adecuadas prefiriendo la via oral hasta donce prosible.

Es necesario que la enferma guarde completo repros. tantr fisico como in. ielectual, lejos de toda clase de preocupaciones que influven de favorablemente sobre su estado general.

\section{Terapia medicanientos:}

Antibióticos. Los más halagadores resultados se har obtenicio con la estreptomicina. Trabajos recientes de Etienne-Bernard, han crmorobadr la perfecta inocuidad que tanto para la madre como para el teto. tierre tha drega (11). No determina trastornos en el dominio del $8^{\circ}$ par craneans de: recten nacido, cuando la madre ha recibido un tratamiento intenso. Su emples an debe ser sistemático 
sino de acuerdo con las indicaciones generales dadas principalmente por la radiografía y el sindrome general. Sin embargo, nos parece aconsejable, cubrir por medio de la estreptomicina, las fases más críticas del desarrollo del embarazo, así: una cura de 2 a 3 meses desde que se hace el diagnóstico de embarazo, una interrupción de 4 a 5 meses durante la fase de remisión y una nueva cura de antibiótico precediendo más o menos 15 días al parto y continuando 1 mes después de él. La dosis que empleamos generalmente es de 1 gmo. cada tercer día por vía intra-muscular, sola o asociada a otros medicamentos, que enunciaremos en seguida.

Acido para-amino-salicilico (P.A.S.). Tiene también muy buenos efectos terapéuticos. Se puede usar solo o asociado a la estreptomicina y se dice que retarda la posibilidad de estreptomicino-resistencia. Los efectos secundarios son iguales a los de los derivados del ácido salicílico, manifestándose principalmente por trastornos gastro-intestinales que desaparecen disminuyendo las dosis.

Derivados del ácido nicotinico. Son substancias para administración oral y de potencia antituberculosa in vitro, mavor que la de cualquier otro agente terapéutico actual. Las dosis excesivas pueden provocar anorexia, excitación del sistema nervioso central, lesion hepática y algunas veces trastornos renales, fenomenos cstos que desaparecen suspendiendo la administración de la droga. Se usan solos o asociados con la estreptomicina y el P.A.S. La dosis media es de $5 \mathrm{Mgm}$. por Kg. de peso, diariamente.

\section{TERAPIA COLAPSANTE:}

Es el factor terapéutico fundamental para esta asociación fisio-patológica. Puede ser mecánico o quirurgicó y toráxico o peritoneal. Creemos que en las embarazadas, durante la primera mitad de su gestación, se debe crear la cámara dérea en el tórax. Conocemos la historia de una enferma con 4 meses de gestatación y con diagnóstico de tuberculosis caseo-neumónica cavitaria derecha y cxudativa productiva izquierda: se le practicó una serie de neumoperitoneos resulares hasta completar (el ultimo de 1.000 c.c.); la enferma empezó a hazer un cuadro de amenaza de aborto; se le hizo el tratamiento indicado durante 1 mes y medio al cabo del cual, abortó un feto muerto y macerado.

Pregunto yo a mi rez: ¿se puede atribuír a la presión aérea intra-abdominal, la muerte de ese feto $y$ su expulsión consecutiva?; es un interrogante que sc presta para practicar estudios posteriores pues en la literatura médica no se encuentra nada al respecto.

Algunos autores han recomendado la frenicectomía e incluso Floyd, juzga, que las enfermas con lesiones bilaterales, soportan mejor la frenicectomía de un ado y el neumotórax del otro, que el neumotórax bilateral. Pavlosky (12), recomienda la frenicectomía cuando existen lesiones cavitarias de la base.

Durante el parto: Consideremos ahora a la enferma en trabajo; una vez iniciado, conviene aislarla del resto de las pacientes para que tenga una vigilancia 
espasmódicos; entre estos últimos nos ha dado gran resultado la eupaverina, pues acorta, de manera apreciable, el periodo de borramiento y dilatación del cuello. Si las contracciones uterinas son demasiado dolorosas, podemos usar sin peligro, los anestésicos por inhalación en forma intermitente; hemos usado el Trilene sin ninguna consecuencia desagradable. Conviene ahorrar cuanto sea posible, los esfuerzos del período expulsivo por el peligro que entraña para la enferma tal demanda de energías. Cremos que está demasiado indicado, la aplicación de fórceps, una vez que se reúnan los requisitos necesarios para hacerlo correctamente. Para esta maniobra, se puede practicar una anestesia en silla de montar que, conservando el estado de lucidez de la paciente da así mismo una gran relajación de la musculatura perineal.

Una vez nacido el niño, debemos atender el alumbramiento en la forma conocida.

La pérdida sanguínea del post-parto debe ser reducida al mínimo. Se debe aplicar 0,2 mgm. de ergotrate intramuscular e igual dosis intravenosa. Si persiste la hemorragia aunque sea insignificante se debe practicar el taponamiento y el masaje uterino.

Cesárea: Se practicará solamente en los casos, en que por su estado pulmonar, se tema algún peligro al dejar trabajar a la enferma (13).

Durante el puerperio. Es la epoca más peligrosa, pues como ya hemos visto, la rápida descompresión torácica puede producir una diseminacion hematógena de la infezción. Es necesario practicar la colapsoterapia artificial; a las parturientas que han tenido su parto por vias naturales, hay que instalarles el neumoperitoneo. Creemos que de 1.000 a $1.500^{\circ}$ c.c. de aire, es suficiente; en nuestro servicio lo practicamos de media a una hora después del parto. En las que se les ha practicado cesárca hay que practicarles neumotorax, por la sencilla razón que la presión del neumoperitoneo puede producir la dehiscencia de la herida quirúrgica. La paciente debe ser estrechamente vigilada. La lactancia debe ser proscrita, pues aunque la mavoría de los autores niegan la transmisión del bacilo por la leche materna, si supone un gran desgaste para el organismo materno, amén del gran peligro de contagio que para el niño significa el acercamiento a su madre.

\section{Profiláxis:}

Si una mujer tiene una tuberculosis cerrada, suele sobrellevar el embarazo sin ningún trastorno; pero debe ser examinada frecuentemente por un internista y llevar una vida sujeta a las mejores condiciones higiénicas.

La actitud será más reservada cuando existan en ella síntomas antiguos como pleuresía, bronquitis repetida, etc.; en estos casos se proscribirán los embarazos repetidos. Si la mujer es una tuberculosa declarada, se desaconsejará francamente el matrimonio. 


\section{Cuidado del Niño:}

Todos los niños deben ser aislados desde el momento de nacer; serán alimentados por nodrizas sanas o por medio de alimentación artificial. Serán vacunados con B.C.G. excepto los prematuros, que lo serán cuando hayan llegado a los 3.000 gmos, de peso.

Es preferible que durante los dos primeros años estén completamente aislados $\left({ }^{1+}\right)$.

Para concluir, damos a conocer una pequeña estadística sobre 30 enfermas que han pasado por el servicio de maternidad del sanatorio de Santa Clara, en el curso de 18 meses que lleva de fundado; estas pacientes pertenecen a varias formas clinicas de tuberculosis pulmonar, que son: caseoneumónica cavitaria, exudativa productiva y detenida.

\begin{tabular}{|c|c|c|}
\hline 19-Primiparas & 5 & $=16,66 \%$ \\
\hline $2^{\circ}-$ Multiparas & 25 & $=83,33 \%$ \\
\hline 39-Caseoneumónicas cavitarias unilaterales . . . . & 14 & $=46,66 \%$ \\
\hline " bilaterales .... & 7 & $=$ \\
\hline 50-Exudativa productiva unilaterales $\ldots . .$. & 6 & $=20 \%$ \\
\hline bilaterales. & 3 & $=10 \%$ \\
\hline 7 -Partos sutócicos $\ldots$ & 19 & $=63$ \\
\hline 8?-Partos distócicos. & 8 & $=26,6$ \\
\hline 90-Cesáreas & 1 & $=0$, \\
\hline $10^{\circ}$-Abortos $\therefore$ & 1 & $=0,3 \%$ \\
\hline$\ldots \ldots \ldots \ldots \ldots$ & 6 & $=20 \%$ \\
\hline $\operatorname{nino} \ldots \ldots \ldots \ldots \ldots \ldots \ldots$ & 23 & $=76,66$ \\
\hline lidad materna . . . . . . . . & 0 & \\
\hline 149-.Mortalidad fetal ... & 1 & $=$ \\
\hline
\end{tabular}

ExPLicación:

Los partos distócicos se presentaron por inercia secundaria, trabajo prolongado " sufrimiento fetal.

Las cesareas, una por desproporción céfalo-pélvica y otra por fibromatosis uierina. Un aborto, cuva única explicación sería el neumoperitoneo repetido que sc le prasticó a la madre.

Los prematuros oscilaron entre $7^{1 / 2}$ a $8 \frac{1}{2}$ meses, pero ninguno con menos de 2.300 gmos. de peso.

Una enferma murió a los 3 días del parto por descompensación cardíaca. 


\section{B I B L I O G R A F I A}

1.-Recasens Girol Sebastián: "Tratado de Obstetricia". (Patología). - 2a Edición. Cap. I. Págs.: 149-411. - Salvat y Cía. Barcelona.

2.-Acosta Hernando: "Conferencias de Obstetricia". - Tomo II. Tesis XIX. Págs.: 84-85-89. - 1951 .

3.-Tapia Manuel: "Formas Anatomo-Clínicas, diagnóstico y tratamiento de la T.B.C. pulmonar”. - Tomo II. Págs.: 264-268. - 2a Edición. Livraria Luso. Espanhola, Lda. Lisboa. Barcelona. - 1946.

4.-Devraigne L.: "Manual de Obstetricia”. - 4a Edición. Cap. II Págs.: 427-428. Espasa Calpe S. A. Madrid. - 1943.

5.-Ramirez Muñoz Carlos. "Acciones mutuas entre tuberculosis y gestación". Tesis de grado. Págs.: 29-37. - 1952.

6.-Nubiola-Zárate: "Tratado de Obstetricia". - Tomo II. Cap. XXII. Pág.: 306. Editorial Labor. Madrid. - 1951.

7.-Botella Llusia José: "Patología Obstétrica". - 1a Edición. Lección 10a . Págs.: 130-132-133. - Editorial Científico-Médica. Barcelona. - 1950.

8. -Goldberg Benjamin: "Clinical Tuberculosis". - 2a Edición. Tomo II. Cap. XL. Págs.: J5-J9. - F. A. Davis Company. - Publishers. Philadelphia. - 1941.

9.-Döderlein A.: “Tratado de Obstetricia”. - 2a Edición. Tomo II. Cap. VI. Pág.: 187. - Editorial Labor S. A. Madrid. - 1938.

10. -De Lee-Greenhill: "Principios y Práctica de Obstetricia". - 7a Edición. Tomo I. Pág.: 621. - Uteha, Méjico.

11.-Bourgeois Pierre-Et-Al: "Techniques et Therapeutiques en Pneumologie". Cap. XXII. Pág.: 222. - L'Expansion Scientifique Francaise. Editeur. París. 1950.

12. - Pérez Manuel Luis: "Tratado de Obstetricia". - 5 a Edición. Volumen 29. Cap. II. Pág.: 117. - Aniceto López. Editor. Buenos Aires. - 1945.

13. - Rey A. J. - Pangas J. C. - Masse R. J.: "Tratado de Tisiologia". - 5a Edición. Cap. XLXIII. Pág.: 514. - Editor "El Ateneo". Buenos Aires. - 1950.

14. - Kayne, Pagel and O'Shaughnessy's: "Pulmonary Tuberculosis". - 2a Edición. Cap. IV. Pág.: 639. - Oxford University Press-London. - 1948. 